\title{
Complex Vascular Reconstruction Following Resection of a Large Retroperitoneal Teratoma
}

\author{
Bo Stüben*1, A Heumann' ${ }^{1}$, S Wipper ${ }^{2}$, P Bannas ${ }^{3}$ and J Li ${ }^{1}$ \\ ${ }^{1}$ Department of General, Visceral and Thoracic Surgery, Germany \\ ${ }^{2}$ Department of Vascular Medicine, Germany \\ ${ }^{3}$ Department of Diagnostic and Interventional Radiology and Nuclear Medicine, Germany
}

*Corresponding author: Björn Ole Stüben MD, Department of General, Visceral and Thoracic Surgery, University Medical

Center Hamburg-Eppendorf, Martinistraße 52, 20246 Hamburg, Germany

\section{ARTICLE INFO}

Received: 彗 January 04, 2021

Published: 輩 January 28, 2021

Citation: BO Stüben, A Heumann, S Wipper, P Bannas, J Li. Complex Vascular Reconstruction Following Resection of a Large Retroperitoneal Teratoma. Biomed J Sci \& Tech Res 33(3)-2021. BJSTR. MS.ID.005412.

Keywords: Germ-Cell Tumor; Malignant Somatic Differentiation; Complex Vascular Reconstruction

\section{ABSTRACT}

Background: Germ cell tumors (GCT) are a common malignancy in males, with variations in differentiation making different therapeutic strategies necessary. Generally, GCT's show good curation rates due to their good response to radiotherapy or chemotherapy. However, certain subtypes are resistant to these therapies and require surgery.

Method: We present a case of a young patient with a large retroperitoneal GCT with somatic malignant transformation, where resection of large abdominal blood vessels with complex reconstruction being necessary to completely remove the tumor.

Results: The tumor was completely resected, and the patient has since been recurrence-free in the follow-up period.

Conclusion: GCT with somatic transformation show high resistance rates to chemoand radiotherapy, and the patient in the presented case study did indeed show only limited response to carboplatin-based chemotherapy. Patients suffering from these conditions should be resected whenever possible, as curation can be achieved by complete tumor resection. Infiltration of neighboring structures is no contraindication to surgery. The case presented here shows that interdisciplinary surgical planning including vascular and general surgeons as well as radiologists is vital to ensure successful tumor resection.

\section{Introduction}

We present a case study of a patient with a large retroperitoneal malignant teratoma and somatic transformation. The patient had previously given consent to the publication of this case. Germ cell tumors (GCT) are the most common solid malignancy in the third and fourth decade in males, with the gonads being the most common localization [1]. The incidence of these diseases has doubled over the last 25 years [2-4].

Extragonadal manifestations are rare, with the mediastinum being the most common site closely followed by the retroperitoneum [5]. GCT show great variation in respect to differentiation, and these variations are important when considering response to therapy. Some tissues are radiosensitive, whilst others respond well to chemotherapy. In general, GCT show good therapeutic response even when discovered in patients with existing metastases, and cure rates of up to $80 \%$ can be achieved [6]. Of these histological subtypes, somatic malignant transformation (SMT) is extremely rare, making up $2 \%$ of all male GCT's [7]. These tumors can histologically resemble any of the 3 germinal layers, sarcomas being the most common subtype $[8,9]$. These tumors have been shown to be resistant to traditional cisplatin-based chemotherapy regimens, and therefore present a therapeutic challenge. Aggressive surgical resection is currently considered the therapy of choice with curation rates of $50-60 \%$ [8-10].

\section{Case Description}

A 25-year-old male patient had been admitted to a regional hospital due to lower abdominal pain. Contrast enhanced computed 
tomography (CT) scan of the abdomen showed a tumor mass of the left psoas major muscle. Subsequently, magnetic resonance imaging (MRI) demonstrated extensive tumor growth in the retroperitoneal space with a tumor size of $>10 \times 10 \mathrm{~cm}$ in diameter with concomitant obstructive uropathy grade III on the left side (Figure 1). The patient was referred to our university medical center for further diagnostics and treatment. During admission, the patient was in good health with a normal BMI. Detailed anamnesis of the patient revealed a weight loss of $3 \mathrm{~kg}$ in the previous 2 months. He also complained of increased perspiration whilst sleeping and fatigue as well as lower abdominal pain. A CT guided biopsy of the tumor mass was performed, the histopathological examination showing a somatic type teratoma. All tumor markers were negative. The multidisciplinary tumor board (MDT) decided for neoadjuvant therapy with carboplatin AUC2 und etoposid followed by $3 \mathrm{x}$ ifosfamide over a period of 3 months.
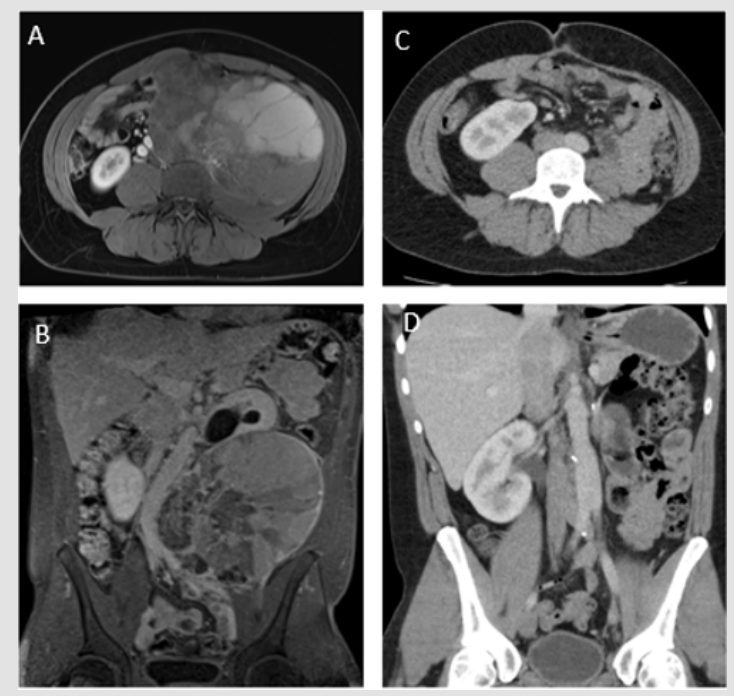

Figure 1:

A. Axial contrast-enhanced MRI, with tumor lesion in the left retroperitoneum;

B. Coronary MRI showing extent of the tumor with left sided uropathy as well as compression of the aorta and the iliac bifurcation;

C. Postoperative axial and coronary contrast-enhanced CT

D. Postoperative axial and coronary contrast-enhanced CT.

The re-staging (FDG-PET/CT) showed only minimal response to therapy and revealed in addition thrombosis of the left common and internal iliac vein. It became apparent that the tumor had extensively infiltrated the retroperitoneum as well as the left ureter, the left common iliac artery, the left iliac vein, and the infrarenal aorta. Therefore, the MDT decided for surgery, leading to an interdisciplinary concept involving the departments of vascular surgery and abdominal surgery. After dissection of the retroperitoneum, the infrarenal aorta as well as both iliacal vessels were crossclamped following intravenous administration of 5000 IU Heparin. The tumor was resected en bloc with the infrarenal aorta, both common iliac arteries, left iliac vein, left kidney and left ureter. In addition, the mesosigmoid, which also showed tumor infiltration, was resected along with the inferior mesenteric artery. An intraoperative florescence examination confirmed good perfusion of the left and sigmoid colon, thus avoiding a segmental colon resection. Subsequently, the infrarenal aorta was replaced by a collagen-, silver-, and triclosan covered bifurcated polyester graft (Intergard Synergy 18/9mm). The left common iliac vein was reconstructed using the previously harvested right common iliac artery and the left renal vein, which had been resected simultaneously during the nephrectomy.

The left renal artery was used to connect the lower pole artery of the right kidney to the aortic graft (Figure 2). Histopathological analyses revealed complete resection (R0) of the tumor, with the tumor showing sarcomatos and carcinomatous differentiation. The tumor was classified as a stage IIIB, intermediate risk (IGCCCG). The postoperative course was uneventful with the patient being discharged after seven days. No edema of the left leg was observed. The renal function was well compensated with serum creatinine in the normal range. Ultrasound exams revealed normal arterial and venous blood flow under therapeutic anticoagulation with lowmolecular heparin (enoxaparin). The enoxaparin was given for six months to prevent venous thrombosis after complex iliac vein reconstruction. Follow-up after one year (thoracoabdominal CT) revealed a normal postoperative status without tumor recurrence. All blood vessels were circulated. 


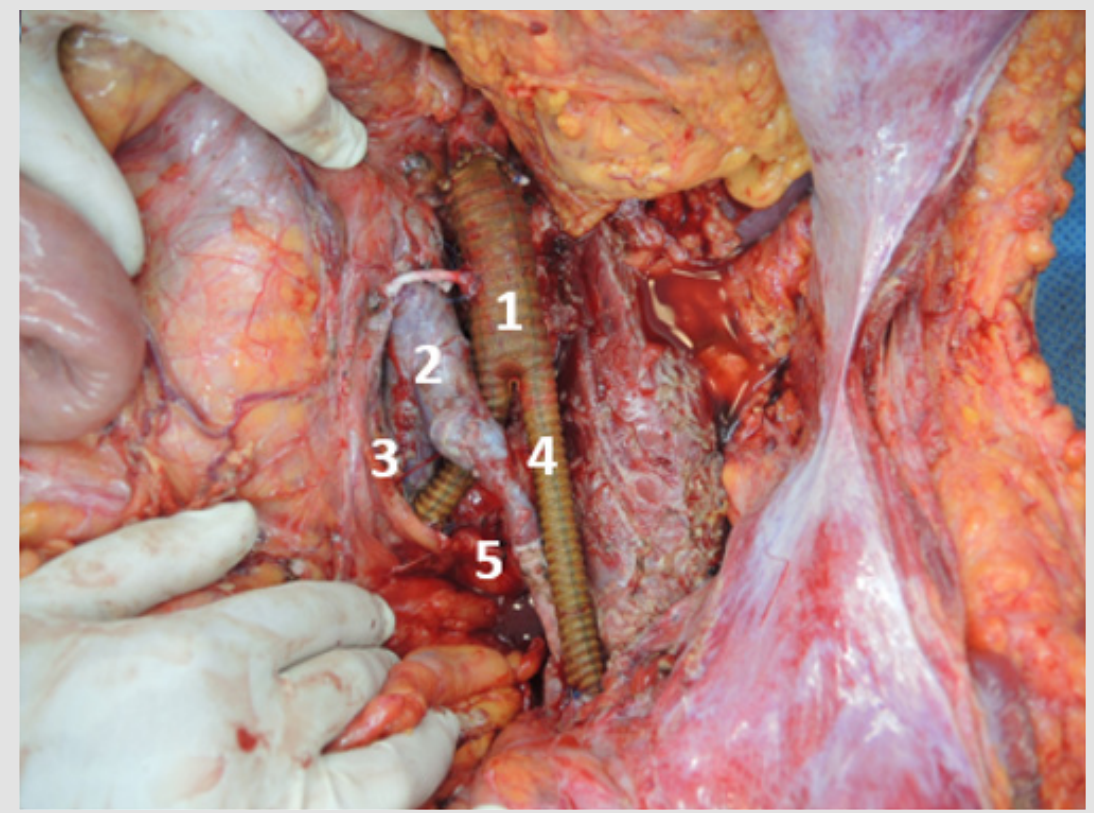

Figure 2: vascular reconstruction following tumor resection:

1. 18/9 Synergy Y-Prosthesis, infrarenal aorta

2. V. cava inferior

3. Right common iliac vein and reconstructed right common iliac artery

4. Reconstructed left common iliac artery

5. Reconstructed left common iliac vein

\section{Discussion}

Whilst GCT generally have a good prognosis with curation rates of $80 \%$ being achievable, somatic differentiation severely impacts prognosis, with significantly lower survival rates of $50-60 \%$ following resection and systemic therapy [6,8-10]. This is largely due to chemoresistance seen in somatically differentiated tumors. Rice et al. demonstrated that patients with somatic differentiation in GCT had a complete response following cisplatin-based chemotherapy of $13 \%$ compared to the expected $70 \%$ which would be seen in metastatic GCT without somatic differentiation [11]. Similarly, to patients with soft tissue sarcomas, patients with GCT and somatic differentiation require surgical resection in order to have acceptable survival chances. Wortmann et al. described 2-year patency rates of arterial bypasses following surgery of soft tissue sarcomas in the retroperitoneum of $88 \%$ [12].

\section{Conclusion}

GCT with somatic transformation show high resistance rates to chemo- and radiotherapy, and the patient in the presented case study did indeed show only limited response to carboplatin-based chemotherapy. Patients suffering from these conditions should be resected whenever possible, as curation can be achieved by complete tumor resection. Infiltration of neighboring structures is no contraindication to surgery. The case presented here shows that interdisciplinary surgical planning including vascular and general surgeons as well as radiologists is vital to ensure successful tumor resection.

\section{Acknowledgement}

A special thank you goes to Sabine Wuttke of the graphics department of the University Medical Center Hamburg-Eppendorf for the drawings shown in Figures $3 \& 4$.
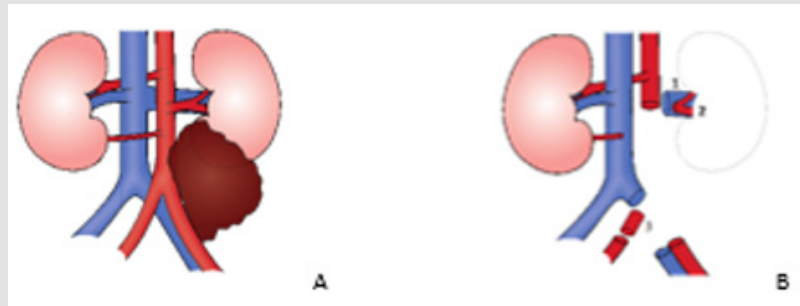

Figure 3: Schematic presentation of the tumor and intraoperative findings

A. Tumor involving the left ureter, infrarenal aorta, the iliac bifurcation, the left iliac artery and left iliac vein

B. The tumor was completely resected with vessels harvested for further reconstruction (1. left renal vein; 2. left renal artery including two segment arteries; 3. right common iliac artery) 

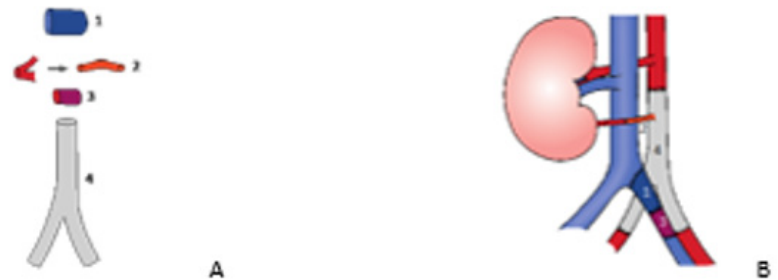

Figure 4: Schematic presentation of the different vascular grafts and reconstruction

A. Overview of the vascular grafts for the venous and arterial reconstruction (1: left renal vein; 2 . left renal artery; 3. Right common iliac artery; 4. 18/9 Synergy Y-prosthesis) B. Complete vascular reconstruction of the infrarenal aorta including iliac bifurcation, the right lower pole renal artery and the left common iliac vein

\section{References}

1. Hayes Lattin B, Nichols CR (2009) Testicular cancer: a prototypic tumor of young adults. Seminars in oncology 36(5): 432-438.

2. Carver BS, Sheinfeld J (2005) Germ cell tumors of the testis. Annals of surgical oncology 12(11):871-880.

3. Mc Kiernan JM, Goluboff ET, Liberson GL, Golden R, Fisch H, et al. (1999) Rising risk of testicular cancer by birth cohort in the United States from 1973 to 1995. J Urol 162(2): 361-363.

4. Purdue MP, Devesa SS, Sigurdson AJ, Mc Glynn KA (2005) International patterns and trends in testis cancer incidence. Int J Cancer 115(5): 822827.

\section{ISSN: 2574-1241}

DOI: $10.26717 /$ BJSTR.2021.33.005412

B0 Stüben. Biomed J Sci \& Tech Res

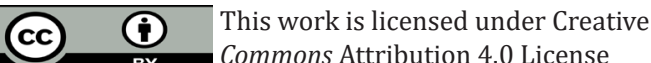

Submission Link: https://biomedres.us/submit-manuscript.php
5. Arora RS, Alston RD, Eden TO, Geraci M, Birch JM, et al. (2012) Comparative incidence patterns and trends of gonadal and extragonadal germ cell tumors in England, 1979 to 2003. Cancer 118(17): 4290-4297.

6. Carver BS, Serio AM, Bajorin D, Motzer RJ, Stasi J, et al. (2007) Improved clinical outcome in recent years for men with metastatic nonseminomatous germ cell tumors. J Clin Oncol 25(35): 5603-5608.

7. Ahmed T, Bosl GJ, Hajdu SI (1985) Teratoma with malignant transformation in germ cell tumors in men. Cancer 56(4): 860-863.

8. Comiter CV, Kibel AS, Richie JP, Nucci MR, Renshaw AA, et al. (1998) Prognostic features of teratomas with malignant transformation: a clinicopathological study of 21 cases. J Urol 159(3): 859-863.

9. Motzer RJ, Amsterdam A, Prieto V, Sheinfeld J, Murty VV, et al. (1998) Teratoma with malignant transformation: diverse malignant histologies arising in men with germ cell tumors. J Urol 159(1): 133-138.

10. Little JS, Foster RS, Ulbright TM, Donohue JP (1994) Unusual neoplasms detected in testis cancer patients undergoing post-chemotherapy retroperitoneal lymphadenectomy. J Urol 152(4): 1144-1149.

11. Rice KR, Magers MJ, Beck SD, Cary KC, Einhorn LH, et al. (2014) Management of germ cell tumors with somatic type malignancy: pathological features, prognostic factors and survival outcomes. J Urol 192(5): 1403-1409.

12. Wortmann M, Alldinger I, Bockler D, Ulrich A, Hyhlik Durr A, et al. (2017) Vascular reconstruction after retroperitoneal and lower extremity sarcoma resection. European journal of surgical oncology: the journal of the European Society of Surgical Oncology and the British Association of Surgical Oncology 43(2): 407-415.

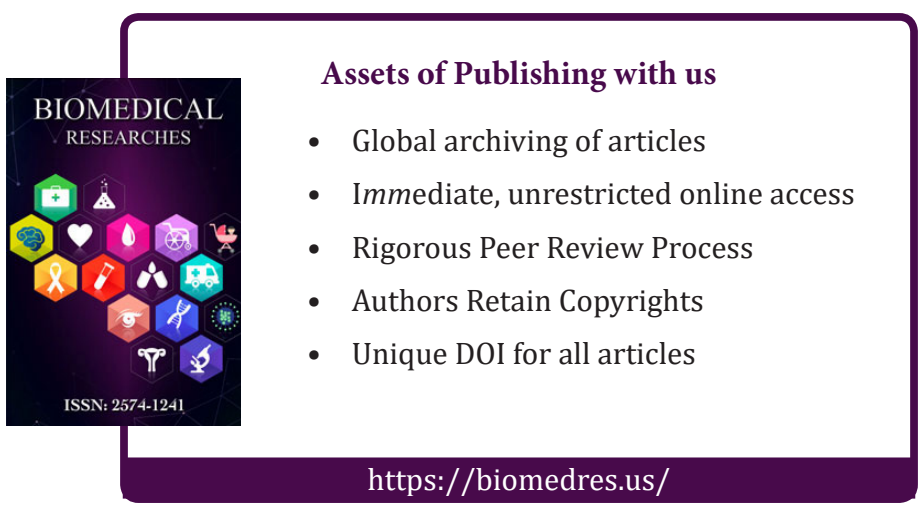

